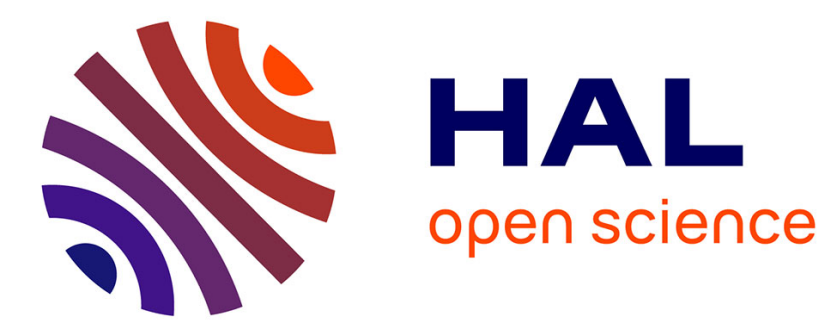

\title{
Evolutionary stability of optimal foraging: partial preferences in the diet and patch models
}

\author{
Vlastimil Křivan
}

\section{To cite this version:}

Vlastimil Křivan. Evolutionary stability of optimal foraging: partial preferences in the diet and patch models. Journal of Theoretical Biology, 2010, 267 (4), pp.486. 10.1016/j.jtbi.2010.09.014 . hal-00637808

\section{HAL Id: hal-00637808 \\ https://hal.science/hal-00637808}

Submitted on 3 Nov 2011

HAL is a multi-disciplinary open access archive for the deposit and dissemination of scientific research documents, whether they are published or not. The documents may come from teaching and research institutions in France or abroad, or from public or private research centers.
L'archive ouverte pluridisciplinaire HAL, est destinée au dépôt et à la diffusion de documents scientifiques de niveau recherche, publiés ou non, émanant des établissements d'enseignement et de recherche français ou étrangers, des laboratoires publics ou privés. 


\section{Author's Accepted Manuscript}

Evolutionary stability of optimal foraging: partial preferences in the diet and patch models

Vlastimil Křivan

PII: S0022-5193(10)00488-1

DOI: doi:10.1016/j.jtbi.2010.09.014

Reference: YJTBI 6154

To appear in:

Journal of Theoretical Biology

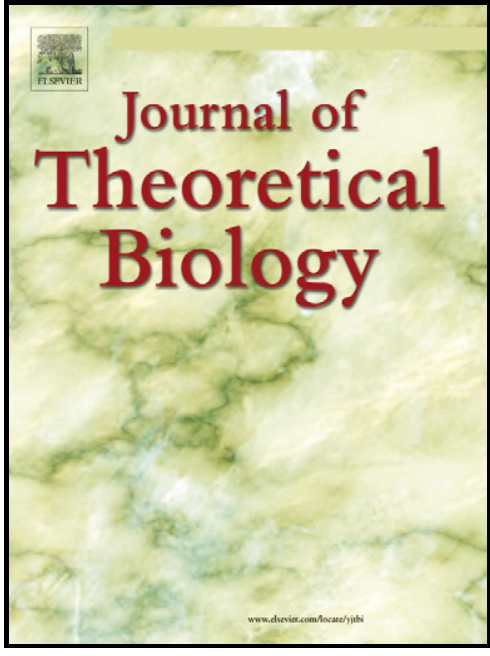

www.elsevier.com/locate/yjtbi

Received date: 16 July 2010

Revised date: $\quad 8$ September 2010

Accepted date: $\quad 9$ September 2010

Cite this article as: Vlastimil Křivan, Evolutionary stability of optimal foraging: partial preferences in the diet and patch models, Journal of Theoretical Biology, doi:10.1016/j.jtbi.2010.09.014

This is a PDF file of an unedited manuscript that has been accepted for publication. As a service to our customers we are providing this early version of the manuscript. The manuscript will undergo copyediting, typesetting, and review of the resulting galley proof before it is published in its final citable form. Please note that during the production process errors may be discovered which could affect the content, and all legal disclaimers that apply to the journal pertain. 


\section{Abstract}

In this article the patch and diet choice models of the optimal foraging theory are re-analyzed with respect to evolutionary stability of the optimal foraging strategies. In their original setting these fundamental models consider a single consumer only and the resulting fitness functions are both frequency and density independent. Such fitness function do not allow us to apply the classical game theoretical methods to study evolutionary stability of optimal foraging strategies for competing animals. In this article frequency and density dependent fitness functions of optimal foraging are derived by separation of time scales in an underlying population dynamical model and corresponding evolutionarily stable strategies are calculated. Contrary to the classical foraging models the results of the present article predict that partial preferences occur in optimal foraging strategies as a consequence of the ecological feedback of consumer preferences on consumer fitness. In the case of the patch occupation model these partial preferences correspond to the Ideal Free Distribution concept while in the case of the diet choice model they correspond to the partial inclusion of the less profitable prey type in predators diet.

Keywords: evolutionarily stable strategy, game theory, ideal free distribution, population growth, predator-prey models 
of such a situation is the experiment with great tits where a single animal feeds on two food types delivered on a conveyor belt (e. g., Krebs et al., 1977; Berec et al., 2003) which assures that prey are not depleted by predation. Thus, no interference or competition for food between consumers is considered. A similar reasoning can be applied to the case where a consumer forages in a heterogeneous environment consisting of discrete foraging patches. In this case the consumer should move to the patch with the highest payoff. These predictions are based on fitness functions that are both density and frequency independent. They consider a single consumer and resource densities that are not influenced by predation. Thus, they miss completely competition between consumers that can be either due to interference, or due to exploitation of resources. In fact, such models can be applied only to laboratory experiments with a single consumer that does not influence resource density (e. g., a single great tit feeding on two prey types delivered on the conveyor belt).

More realistic models must consider consumer competition (either interference competition, or exploitative competition). A density dependent approach in patchy environments led to the concept of the Ideal Free Distribution (IFD; Fretwell and Lucas, 1969). These authors assumed that patch payoff decreases with increasing number of individuals in that patch (e. g., this captures interference among birds) and predicted that under the IFD no individual can increase its fitness by changing its strategy measured by residence times in different patches. Because under the IFD all patches provide animals with the same fitness the question arises whether such a situation is stable or not. Indeed, assuming a single mutant with an infinitely large resident population that is distributed according to the IFD, fitness of that mutant is independent and the same as is the fitness of resident animals. One approach to study stability in such situations is to use the concept of evolutionarily stable strategies (Maynard Smith and Price, 1973; Hofbauer and Sigmund, 1998). In fact it has been shown that the IFD is an evolutionarily stable strategy of the habitat selection game (Krivan et al., 2008). However, this concept of stability assumes that the fitness function is frequency dependent, i. e., it depends both on the mutant as well as on the resident strategy. Frequency dependence allows us to predict whether a mutant strategy will increase in the resident population or not. Here the idea is that the resident strategy sets the environment (e. g., the resident consumer strategy sets the resource densities) and if a mutant strategy with a higher fitness exists then this strategy spreads, replaces residents, sets a new environment and so on until an evolutionarily stable strategy (ESS) is reached.

Another approach that considers explicitly resource-consumer population dynamics together with optimal foraging was also used in theoretical ecology (reviewed in Abrams, 2010). These models show how resident individuals that follow optimal foraging strategy influence resource levels which, in turn, influence consumer numbers and strategy. Although dynamical models capture the ecological feedback, they do not provide us with information about the evolutionary stability of optimal foraging strategies. To study this question it is necessary to study conditions under which the optimal foraging strategy is resistant to mutant invasions. Studying evolutionary stability in dynamic models of optimal foraging is more complicated than in static 
models of behavioral ecology (such as the Hawk-Dove model, or the Prisoners dilemma; Maynard Smith and Price, 1973; Hofbauer and Sigmund, 1998) because it requires to analyze population dynamics for both resources, resident and mutant consumers (e. g., Křivan and Cressman, 2009; Cressman and Křivan, 2010).

To sum up, the problem is that some models of optimal foraging are described by fitness functions that are both frequency and density independent, and they do not allow us to predict optimal foraging strategies when interference or resource depletion occur. On the other hand, in the full dynamic setting that describes explicitly the effect of resident strategies on resource densities, the resulting models are multidimensional and difficult to analyze with respect to evolutionary stability of optimal strategies. My aim is to show how frequency dependent fitness functions can be obtained from frequency independent fitness functions, using a time scale argument. I start with a frequency independent fitness function defined as the per capita population growth rate. Then I consider resource-consumer population dynamics and I assume that resource dynamics run on a faster time scale when compared with consumer population dynamics. This is the case of many resource-consumer systems where resource generation time is shorter than consumer generation time (e. g., many plants have short generation times relative to mammalian herbivores). This time scale separation allows me to assume that at each consumer density resources are at the corresponding population equilibrium. Substituting this resource equilibrium to the consumer fitness function leads to a frequency dependent fitness function that can be analyzed from evolutionary perspective. I apply this approach to diet and patch choice paradigms of optimal foraging theory. In both cases this approach predicts emergence of partial preferences for alternative prey/patch types. In the case of the patch model partial preferences describe the consumer Ideal Free Distribution (Fretwell and Lucas, 1969). Although partial preferences are not predicted by the classical frequency independent models of optimal foraging, they were observed in many foraging studies (for a review see Stephens and Krebs, 1986). Explanations for partial preferences range from incorrect classification of resource and sampling by consumers (Krebs et al., 1977; Rechten et al., 1983), resource crypsis (Erichsen et al., 1980), incorrect estimation of encounter rates with resource by consumers (McNamara and Houston, 1987; Hirvonen et al., 1999), limited memory capacity of consumers (Mangel and Roitberg, 1989; Bélisle and Cresswell, 1997), and limited knowledge of the environment (Berec and Křivan, 2000). In this article I will show another mechanism that explains emergence of partial preferences by the ecological feedback of consumer preferences on their fitness.

\section{The patch occupation model}

I will consider an environment consisting of two foraging patches containing resources with abundance $x_{i}$ in patch $i(i=1,2)$. If $e_{i}$ denotes the efficiency with which resources are converted to new consumers, $\lambda_{i}$ is the consumer search rate for resources in patch $i$, and $m_{i}$ is the consumer mortality rate in patch $i$ then consumer fitness expressed as the instantaneous per capita population growth rate (cf. consumer per 
capita population growth rate in model (3) below) is

$$
W=\left(e_{1} \lambda_{1} x_{1}-m_{1}\right) v_{1}+\left(e_{2} \lambda_{2} x_{2}-m_{2}\right) v_{2}
$$

where $v_{i}\left(v_{1}+v_{2}=1\right)$ is the proportion of the time a consumer stays in patch $i$. Thus, $v_{i}$ measures the consumer preference for patch $i$. Assuming that consumer preferences are adaptive, the optimal consumer strategy is to stay in patch 1 when patch payoff is higher there than in patch 2 and vice versa. Thus, the strategy that maximizes consumer fitness $W$ is

$$
v_{1}=\left\{\begin{array}{lll}
1 & \text { if } e_{1} \lambda_{1} x_{1}-m_{1}>e_{2} \lambda_{2} x_{2}-m_{2} \\
0 & \text { if } e_{1} \lambda_{1} x_{1}-m_{1}<e_{2} \lambda_{2} x_{2}-m_{2} .
\end{array}\right.
$$

This simple prediction states that consumers should move to the patch that provides them with the highest fitness. It is clear that mutants using a different strategy obtain a lower fitness and therefore, the above strategy is evolutionarily stable. However, due to interference or resource depletion it is also obvious that as more and more consumers move to a patch, this patch payoff must decrease, which is not captured by maximization of fitness function $W$. To model effects of consumers on resources, I will consider the following population dynamics (e. g., Fryxell and Lundberg, 1994, 1997; Křivan, 1997; Křivan and Schmitz, 2003)

$$
\begin{aligned}
\frac{d x_{1}}{d t} & =a_{1} x_{1}\left(1-\frac{x_{1}}{L_{1}}\right)-v_{1} \lambda_{1} x_{1} y \\
\frac{d x_{2}}{d t} & =a_{2} x_{2}\left(1-\frac{x_{2}}{L_{2}}\right)-v_{2} \lambda_{2} x_{2} y \\
\frac{d y}{d t} & =\left(e_{1} \lambda_{1} x_{1}-m_{1}\right) v_{1} y+\left(e_{2} \lambda_{2} x_{2}-m_{2}\right) v_{2} y
\end{aligned}
$$

where $y$ is consumer density, $L_{i}$ is the resource $i$ carrying capacity, and $a_{i}$ is the resource per capita population growth rate. I stress here that consumer fitness (1) is indeed the per capita consumer population growth rate in model (3).

Using model (3) I derive a new frequency dependent fitness function. I consider a mutant with a strategy $\tilde{v}=\left(\tilde{v}_{1}, \tilde{v}_{2}\right)$ in a population of residents with a strategy $v=\left(v_{1}, v_{2}\right)$ and I re-write frequency independent fitness function (1) as a function of both mutant and resident strategies

$$
W(\tilde{v}, v)=\left(e_{1} \lambda_{1} x_{1}-m_{1}\right) \tilde{v}_{1}+\left(e_{2} \lambda_{2} x_{2}-m_{2}\right) \tilde{v}_{2}
$$

The dependence of this function on the resident strategy $\left(v=\left(v_{1}, v_{2}\right)\right)$ is indirect through resource abundance that is set by the resident strategy in model (3). To make this dependence explicit I will assume that resource population dynamics run on a fast time scale when compared with consumer population dynamics so that for each consumer density resources relatively quickly reach their corresponding equilibrium 
densities. When residents use strategy $v=\left(v_{1}, v_{2}\right)$, the resource equilibrium at consumer density $y$, calculated from (3), is $x_{i}=L_{i}\left(1-\lambda_{i} v_{i} y / a_{i}\right),(i=1,2)$. Substituting this density in $W$ leads to a frequency dependent fitness function

$$
W(\tilde{v}, v)=r_{1} \tilde{v}_{1}\left(1-\frac{v_{1} y}{K_{1}}\right)+r_{2} \tilde{v}_{2}\left(1-\frac{v_{2} y}{K_{2}}\right)
$$

where

$$
r_{i}=e_{i} \lambda_{i} L_{i}-m_{i} \text { and } K_{i}=\frac{a_{i}\left(e_{i} \lambda_{i} L_{i}-m_{i}\right)}{e_{i} L_{i} \lambda_{i}^{2}}
$$

are the per capita consumer population growth rate and the environmental carrying capacity in patch $i$. It is an interesting observation that the new frequency dependent fitness function is described by logistic consumer population growth in each patch. In other words, if we assume that consumer population growth in each patch is logistic, we would arrive immediately to fitness function (4). Evolutionarily stable strategy for fitness function $W$ was derived by Cressman et al. (2004) and I briefly recall main results here. Assuming that $r_{1}>r_{2}$, there are two possibilities. Either consumer density is low $\left(y<\left(r_{1}-r_{2}\right) K_{1} / r_{1}\right)$ in which case only the better patch 1 will be occupied, or when consumer population is above the critical threshold then both patches will be occupied by consumers, patch payoffs will be the same and the corresponding ESS is

$$
v_{1}^{*}=\frac{K_{1} r_{2}}{K_{1} r_{2}+K_{2} r_{1}}+\frac{K_{1} K_{2}\left(r_{1}-r_{2}\right)}{\left(K_{1} r_{2}+K_{2} r_{1}\right) y} .
$$

Under this strategy no individual can increase its fitness by changing its strategy and this strategy cannot be invaded by individuals using a different strategy. This shows that when patch payoffs are the same the ESS depends on the consumer population density. This ESS corresponds to the IFD of Fretwell and Lucas (1969). When consumers re-distribute instantaneously according to the IFD at each consumer density, consumer population growth is described by a piecewise logistic equation$$
\frac{d y}{d t}= \begin{cases}r_{1} y\left(1-\frac{y}{K_{1}}\right) & \text { if } y \leq \frac{\left(r_{1}-r_{2}\right) K_{1}}{r_{1}} \\ \frac{r_{1} r_{2}\left(K_{1}+K_{2}\right)}{K_{2} r_{1}+K_{1} r_{2}} y\left(1-\frac{y}{K_{1}+K_{2}}\right) & \text { if } y>\frac{\left(r_{1}-r_{2}\right) K_{1}}{r_{1}},\end{cases}
$$

(Křivan and Sirot, 2002). The equilibrium of this model is $K_{1}+K_{2}$ and at this equilibrium consumer preferences satisfy

$$
\frac{v_{1}^{*}}{v_{2}^{*}}=\frac{K_{1}}{K_{2}}
$$

These preferences correspond to the so called balanced dispersal under which no mutants can invade (McPeek and Holt, 1992; Padrón and Trevisan, 2006; DeAngelis et al., 2007). 
Now I compare solutions of the resource-consumer model (3) (Figure 1, solid lines) with optimal consumer strategy given by (2), with solutions of the single-species logistic model (7) (Figure 1, dashed lines). Křivan and Schmitz (2003) showed that for $L_{i}>m_{i} /\left(e_{i} \lambda_{i}\right)$, consumer population dynamics described by (3) converge on the equilibrium density $K_{1}+K_{2}$ at which the corresponding IFD is given by formula (8) exactly as for model (7). This means that model (7) with optimal strategy (6) converges on the same equilibrium as the original resource-consumer model (3) (Figure 1). In addition, if resource population dynamics are much faster than consumer population dynamics (Figure 1, left panel), population trajectories of the reduced model (7) are close to trajectories of the original model (3). If both resource and consumer population dynamics operate on comparable time scales (Figure 1, right panel) the differences between trajectories get larger.

\section{The diet choice model}

Now I will consider the diet choice model. Assuming two prey types, consumer fitness in a fine-grained environment is

$$
\frac{e_{1} \lambda_{1} u_{1} x_{1}+e_{2} \lambda_{2} u_{2} x_{2}}{1+h_{1} \lambda_{1} u_{1} x_{1}+h_{2} \lambda_{2} u_{2} x_{2}}
$$

(Charnov, 1976). Here $x_{i}$ is density of resource $i$ in the environment, $\lambda_{i}$ is the consumer search rate for prey type $i, u_{i}$ is the consumer preference for prey type $i\left(0 \leq u_{i} \leq 1\right)$, $h_{i}$ is the handling time, and $e_{i}$ is the net energy gain obtained from prey type $i$. I stress here that on the contrary to the patch model, the diet choice model does not assume that the sum of strategies equals to one. Optimal foraging theory assumes that resource densities are fixed, and maximization of (9), with respect to consumer preferences for either resource, leads to the zero-one rule (Charnov, 1976). This rule predicts that consumers will always forage on the most profitable resource type, i. e., the resource type with the highest ratio $e_{i} / h_{i}$. In what follows I will assume that the resource type 1 is more profitable than the alternative resource type 2 (i. e., $e_{1} / h_{1}>e_{2} / h_{2}$ ). Therefore, resource 1 will be always included in the consumer diet $\left(u_{1}=1\right)$ while the second resource type will be included only if density of the first resource decreases below the threshold density

$$
x_{1}^{*}=\frac{e_{2}}{\lambda_{1}\left(e_{1} h_{2}-e_{2} h_{1}\right)},
$$

(e. g., Charnov, 1976; Stephens and Krebs, 1986). Consumer strategy is not uniquely predicted by the diet choice model when the more profitable prey density equals $x_{1}^{*}$, because under this condition any strategy will lead to the same fitness.

Now I will derive a frequency dependent fitness function and find the corresponding ESS. For this I consider ecological feedback between consumers and resources that is described by the following Rosenzweig-MacArthur predator-prey dynamics (Křivan 
and Schmitz, 2003)

$$
\frac{d x_{1}}{d t}=a_{1} x_{1}\left(1-\frac{x_{1}}{L_{1}}\right)-\frac{\lambda_{1} x_{1} y}{1+h_{1} \lambda_{1} x_{1}+h_{2} \lambda_{2} u_{2} x_{2}}
$$

$$
\frac{d y}{d t}=\left(\frac{e_{1} \lambda_{1} x_{1}+e_{2} \lambda_{2} u_{2} x_{2}}{1+h_{1} \lambda_{1} x_{1}+h_{2} \lambda_{2} u_{2} x_{2}}-m\right) y .
$$

This model considers population dynamics of the preferred resource $\left(x_{1}\right)$ and consumers $(y)$. It assumes that the alternative resource type density $x_{2}$ stays constant. This can be the case where there is an influx of alternative food resources to the system from outside sources (allochthonous resource; Huxel and McCann, 1998). Parameter $u_{2}$ describes consumer preferences for the alternative resource.

Fitness of a mutant consumer with strategy $\tilde{u}_{2}$ in a population of residents with strategy $u_{2}$ is

$$
W\left(\tilde{u}_{2}, u_{2}\right)=\frac{e_{1} \lambda_{1} x_{1}+e_{2} \lambda_{2} \tilde{u}_{2} x_{2}}{1+h_{1} \lambda_{1} x_{1}+h_{2} \lambda_{2} \tilde{u}_{2} x_{2}}
$$

and I derive a frequency dependent fitness function as in the case of the patch model. For each fixed consumer strategy the resource 1 isocline (given by $d x_{1} / d t=0$ ) is an upside down parabola (Figure 2). Assuming that resource dynamics run on a fast time scale when compared with consumer demography, trajectories move in the direction of the arrows in Figure 2 and they quickly reach the stable part of the resource isocline (solid line)

$$
x_{1+}=\frac{-1+h_{1} L_{1} \lambda_{1}-h_{2} u_{2} x_{2} \lambda_{2}+H\left(u_{2}\right) \sqrt{1-c\left(u_{2}\right) y}}{2 h_{1} \lambda_{1}}
$$

where

$$
H\left(u_{2}\right)=1+h_{1} L_{1} \lambda_{1}+h_{2} x_{2} u_{2} \lambda_{2}, \quad c\left(u_{2}\right)=\frac{4 h_{1} L_{1} \lambda_{1}^{2}}{a_{1} H^{2}\left(u_{2}\right)} .
$$

I remark that the resource isocline is defined only for consumer densities that satisfy $y<1 / c\left(u_{2}\right)$ which I will assume below. Substituting the equilibrium resource density $x_{1+}$ in (12) leads to the frequency dependent fitness function

$$
W\left(\tilde{u}_{2}, u_{2}\right)=\frac{2 h_{1}\left(e_{1} L_{1} \lambda_{1}+e_{2} x_{2} \tilde{u}_{2} \lambda_{2}\right)-e_{1} H\left(u_{2}\right)\left(1-\sqrt{1-c\left(u_{2}\right) y}\right)}{2 h_{1} H\left(\tilde{u}_{2}\right)-h_{1} H\left(u_{2}\right)\left(1-\sqrt{1-c\left(u_{2}\right) y}\right)} .
$$

The selection gradient $\frac{\partial W}{\partial \tilde{u}_{2}}\left(\tilde{u}_{2}, u_{2}\right)$ predicts the direction of selective pressure (Vincent and Brown, 2005; Dercole and Rinaldi, 2008). When the gradient is positive, preference for the alternative prey type will increase, while if it is negative, preference will decrease. The gradient is zero at the singular strategy (Appendix A)

$$
u_{2}^{*}=\frac{L_{1} \lambda_{1}^{2}\left(e_{1} h_{2}-e_{2} h_{1}\right)}{a_{1} h_{2} \lambda_{2} x_{2}\left(L_{1} \lambda_{1}\left(e_{1} h_{2}-e_{2} h_{1}\right)-e_{2}\right)} y-\frac{e_{1}}{\lambda_{2} x_{2}\left(e_{1} h_{2}-e_{2} h_{1}\right)}
$$


provided this value is between zero and one, i. e., consumer density satisfies $y_{1}^{*} \leq y \leq y_{2}^{*}$ where

$$
y_{1}^{*}=\frac{a_{1} e_{1} h_{2}\left(L_{1} \lambda_{1}\left(e_{1} h_{2}-e_{2} h_{1}\right)-e_{2}\right)}{\left(e_{2} h_{1}-e_{1} h_{2}\right)^{2} L_{1} \lambda_{1}^{2}}
$$

and

$$
y_{2}^{*}=\frac{a_{1} h_{2}\left(L_{1} \lambda_{1}\left(e_{1} h_{2}-e_{2} h_{1}\right)-e_{2}\right)\left(e_{1}+\left(e_{1} h_{2}-e_{2} h_{1}\right) x_{2} \lambda_{2}\right)}{\left(e_{2} h_{1}-e_{1} h_{2}\right)^{2} L_{1} \lambda_{1}^{2}}
$$

from Appendix A. For consumer densities for which $y<y_{1}^{*}$, I set $u_{2}^{*}=0$ and for $y>y_{2}^{*}$, I set $u_{2}^{*}=1$. Figure $3 \mathrm{~A}$ shows dependence of the singular strategy on consumer density. It is proved in Appendix A that the singular strategy is not only evolutionarily stable but also convergence stable (Eshel, 1983; Eshel et al., 1997). This means that (1) the singular strategy is resistant to invasion of a small number of mutants that use a different strategy, and (2) it is also resistant to changes in the resident strategy (i. e., when the resident strategy is slightly perturbed, it will return to the convergence stable singular point). Strategies that are evolutionarily and convergence stable are thought as the end point of evolution (Vincent and Brown, 2005; Dercole and Rinaldi, 2008).

Now I will analyze the singular consumer strategy as a function of consumer density. If resource 1 carrying capacity is low so that

$$
L_{1}<x_{1}^{*}
$$

(where $x_{1}^{*}$ is given by (10)) consumers will include the alternative prey type in their diet $\left(u_{2}^{*}=1\right)$ at all consumer densities, because the critical thresholds $y_{1}^{*}$ and $y_{2}^{*}$ are negative. Because $x_{1}^{*}$ is the critical threshold predicted by the optimal foraging theory, below which the alternative prey type is included in the consumers' diet, the present model predicts that at low environmental carrying capacities for the preferred prey type consumers will behave as generalists. This is because at such low carrying capacities the density of the more profitable prey type can never be higher than is the switching threshold $x_{1}^{*}$.

When the carrying capacity of the preferred resource is higher than is the switching density (i. e., the inequality in (16) is reversed), $u_{2}^{*}$ is a piecewise linear function of the consumer density $y$ (Figure 3A). In this case at low consumer densities consumers will behave as specialists feeding on the more profitable resource type only. As the number of consumers increases resource 1 density will decrease and consumers will start to feed on the alternative resource as well, and their preferences for this resource will increase with consumer numbers. When at high numbers consumers will feed on the alternative food items upon each encounter. This shows that when the consumer fitness reflects frequency and density dependence given by the ecological feedback, partial preferences do arise in the diet choice model.

Now I will consider consumer population dynamics. Consumer dynamics along the stable branch of the prey isocline are obtained by substituting expression for $x_{1+}$ in the consumer equation in (11). This leads to the following consumer population growth 
equation

$$
\frac{d y}{d t}=r y\left(1-\frac{K\left(u_{2}\right)}{1+\sqrt{1-c\left(u_{2}\right) y}}\right)
$$

where $r=\frac{e_{1}}{h_{1}}-m$ and $K\left(u_{2}\right)=2 \frac{e_{1}+\left(e_{1} h_{2}-e_{2} h_{1}\right) x_{2} \lambda_{2} u_{2}}{H\left(u_{2}\right)\left(e_{1}-h_{1} m\right)}$ (Appendix B). Substituting for $u_{2}$ in model (17) the singular strategy $\left(u_{2}^{*}\right)$, describes consumer dynamics with optimal diet selection. For consumer densities for which the consumer singular strategy $u_{2}^{*}$ is between 0 and 1 , consumer population dynamics simplify to

$$
\frac{d y}{d t}=y \frac{e_{2}-h_{2} m}{h_{2}}
$$

Depending on the sign of $e_{2}-h_{2} m$, consumer density either increases or decreases when partial preferences occur. Because the above equation has no non-trivial population equilibrium it follows that no population equilibrium where consumers would show partial preferences for the alternative prey type exists. Consumer equilibria for consumer densities that are higher than $y_{2}^{*}$ (i. e., when $u_{2}^{*}=1$ ) or smaller than $y_{1}^{*}$ (i. e., when $u_{2}^{*}=0$ ) are given by the interior equilibrium of $(17) y^{*}=\frac{K\left(u_{2}\right)\left(2-K\left(u_{2}\right)\right)}{c\left(u_{2}\right)}$ where I substitute 0 or 1 for $u_{2}$ (these are given explicitly in Appendix B). Figure 3B shows one such trajectory of model (17) driven by the consumer singular strategy (solid lines). The corresponding resource 1 density is calculated from (13). Figure 3C shows the corresponding consumer singular strategy. These results predict that at low consumer densities where resource 1 density is near to its carrying capacity, consumers specialize on the more profitable resource type. As consumers increase in numbers, the preferred resource density decreases to the critical switching density $\left(x_{1}^{*}\right)$ predicted by the classical model of optimal foraging. From then on, consumer preferences for the alternative prey type start to increase, keeping the preferred resource density at the switching threshold for some time (i. e., times approx. between 5 and 14 in Figure 3B). Because $e_{2}-m h_{2}>0$ in Figure 3B, consumer density increases (it would decrease otherwise). Once the consumer preference reaches 1 (i. e., consumers attack the alternative resource upon each encounter), consumers and resources tend to a stable equilibrium. This general pattern of population dynamics is also clearly shown in Figure 3D where the trajectory from panel $\mathrm{B}$ is projected on the consumer isocline (the solid dot denotes the population equilibrium). Thus, in the region of consumer densities where preferences for the less profitable resource type are between 0 and 1, consumers exhibit partial preferences. This is a new result because the classical diet choice model does not predict such a gradual shift in consumer preferences at the switching prey density (Stephens and Krebs, 1986). Trajectories of model (11) with the optimal consumer foraging strategy are shown in Figure 3B as dashed lines. It is clear that they converge to the same equilibrium as trajectories of the single species model. Using a completely different approach Křivan (1996) (see formula (32) there) calculated consumer strategy at the switching threshold $x_{1}=x_{1}^{*}$ (i. e., when the classical diet choice model does not define consumer preference for the alternative prey type uniquely). It is an interesting 
observation that the formula for partial preferences obtained there coincides with the singular strategy $u_{2}^{*}$.

\section{Discussion}

In this article I have shown how to derive a frequency dependent fitness function from a frequency independent fitness function by using a time-scale argument. The time scale argument assumes that resource population dynamics run on a faster time scale when compared with consumer population dynamics. Having a frequency dependent fitness function allowed me to study optimal foraging strategies for multiple consumers and depletable resources. In particular I showed that partial preferences for alternative resources arise in the diet and patch models of optimal foraging.

The diet and patch choice models are two paradigms of the optimal foraging theory (MacArthur and Pianka, 1966; Emlen, 1966). They assume that per capita energy intake rate is a proxy for consumer fitness that is maximized by adaptive consumer foraging behavior. The diet choice model assumes that two or more resources are evenly distributed in the environment and consumption of these resources does not influence their numbers. This assumption is clearly violated in most natural systems. To comply with these assumptions, e. g., conveyor belts that bring food directly to consumers were used in experiments (Krebs et al., 1977; Berec et al., 2003). So what is missing in the diet choice model is the link between consumer numbers and consumer strategy. This link defines an ecological feedback mechanism: consumer foraging strategy influences resource densities which, in turn, set the consumer strategy and density. To model this ecological feedback mechanism some authors considered population dynamics together with the optimal foraging models (e. g., Fryxell and Lundberg, 1994; Krrivan, 1996; Fryxell and Lundberg, 1997; Křivan, 1997; Křivan and Schmitz, 2003; Ma et al., 2003). In these models it often happens that population dynamics tend to densities at which models of optimal foraging do not predict the optimal strategy uniquely. For example, in the patch model consumers will distribute between the two patches so that patch payoffs will equalize (Krrivan, 1997). Under this situation there is no selection against mutants that can use any strategy, because individual fitness is independent of the strategy. Similarly, dynamical models of diet choice drive periodically density of the more profitable prey type to the threshold where optimal consumer diet is not uniquely predicted (Křivan, 1996). In fact, this non-uniqueness causes fundamental problems for the existence of solutions to these population models (Colombo and Krrivan, 1993; Krrivan, 1996).

A simpler approach to study evolutionary stability is based on the concept of evolutionarily stable strategies (e. g., Maynard Smith and Price, 1973; Hofbauer and Sigmund, 1998; Cressman, 2003; Vincent and Brown, 2005). However, to apply methods of static game theory, the fitness function must be frequency dependent, i. e., it must allow us to measure the effect of mutants on the resident strategy. In this article I derived two frequency dependent fitness functions for patch and diet choice models. These fitness functions are based on the assumption that resource population dynamics 
run on a faster population time scale when compared with consumer population dynamics. Using these fitness functions I derived the corresponding evolutionarily stable strategies for the patch and the diet choice model. In both cases the corresponding ESS predicts partial preferences for resources to occur. In the case of the patch model separation of the resource and consumer time scales leads to consumer population growth that is described by the logistic equation for which the evolutionarily stable strategies were studied (e. g., Křivan and Sirot, 2002; Morris, 2003; Cressman and Křivan, 2006). These works show that the evolutionarily stable strategy corresponds with the IFD (Fretwell and Lucas, 1969). Similarly, when time scaling is applied to the diet choice model the resulting consumer population growth equation seems to be new. Moreover, partial preferences arise for a range of consumer densities in both models. This is a new prediction because partial preferences were not predicted by the optimal foraging theory (Charnov, 1976). In fact, my present analysis clearly shows that at low consumer densities consumers should specialize on the more profitable prey/patch type only. However, as consumer density increases, consumers also start to utilize the alternative, less profitable prey/patch type with increasing strength. This prediction should be easily tested using appropriate data on consumer preferences.

These predictions may have several consequences in population ecology. For example, if consumer preferences depend on consumer numbers, functional responses used in multiple species models should reflect this situation. Thus, they should depend not only on resource densities, but also on consumer densities (for a recent review of flexible foraging on the functional response see Abrams, 2010). Such a dependency could lead to a more mechanistic explanation of the effect of consumer density on the functional response.

\section{Acknowledgments}

The Institute of Entomology is funded by the Academy of Sciences of the Czech Republic (Z50070508). A part of this work was conducted while a Sabbatical Fellow at the National Institute for Mathematical and Biological Synthesis, an Institute sponsored by the National Science Foundation, the U.S. Department of Homeland Security, and the U.S. Department of Agriculture through NSF Award \#EF-0832858, with additional support from The University of Tennessee, Knoxville. 


\section{References}

Abrams, P. A., 2010. Implications of flexible foraging for interspecific interactions: lessons from simple models. Functional Ecology 24, 7-17.

Bélisle, C., Cresswell, J., 1997. The effects of a limited memory capacity on foraging behavior. Theoretical Population Biology 52, 78-90.

Berec, L., Křivan, V., 2000. A mechanistic model for partial preferences. Theoretical Population Biology 58, 279-289.

Berec, M., Křivan, V., Berec, L., 2003. Are great tits (Parus major) really optimal foragers? Canadian Journal of Zoology 81, 780-788.

Charnov, E. L., 1976. Optimal foraging: attack strategy of a mantid. American Naturalist $110,141-151$.

Colombo, R., Křivan, V., 1993. Selective strategies in food webs. IMA Journal of Mathematics Applied in Medicine and Biology 10, 281-291.

Cressman, R., 2003. Evolutionary Dynamics and Extensive Form Games. The MIT Press, Cambridge MA.

Cressman, R., Křivan, V., 2006. Migration dynamics for the ideal free distribution. The American Naturalist 168, 384-397.

Cressman, R., Křivan, V., 2010. The ideal free distribution as an evolutionarily stable state in density-dependent population games. Oikos 119, 1231-1242.

Cressman, R., Křivan, V., Garay, J., 2004. Ideal free distributions, evolutionary games, and population dynamics in multiple-species environments. American Naturalist 164, $473-489$.

DeAngelis, D. L., Vos, M., Mooij, W. M., Abrams, P. A., 2007. Feedback effects between the food chain and induced defense strategies. In: Rooney, N., McCann, K. S., Noakes, D. L. G. (Eds.), From energetics to ecosystems: The dynamics and structure of ecological systems. Springer, Heidelberg, pp. 213-235.

Dercole, F., Rinaldi, S., 2008. Analysis of evolutionary processes. The adaptive dynamics approach and its applications. Princeton University Press, Princeton.

Emlen, J. M., 1966. The role of time and energy in food preferences. American Naturalist $100,611-617$.

Erichsen, J. T., Krebs, J. R., Houston, A. I., 1980. Optimal foraging and cryptic prey. Journal of Animal Ecology 49, 271-276. 
Eshel, I., 1983. Evolutionary and continuous stability. Journal of Theoretical Biology 103, 99-111.

Eshel, I., Motro, U., Sansone, E., 1997. Continuous stability and evolutionary convergence. Journal of Theoretical Biology 185, 333-343.

Fretwell, D. S., Lucas, H. L., 1969. On territorial behavior and other factors influencing habitat distribution in birds. Acta Biotheoretica 19, 16-32.

Fryxell, J. M., Lundberg, P., 1994. Diet choice and predator-prey dynamics. Evolutionary Ecology 8, 407-421.

Fryxell, J. M., Lundberg, P., 1997. Individual behavior and community dynamics. Chapman \& Hall, London, UK.

Hirvonen, H., Ranta, E., Rita, H., Peuhkuri, N., 1999. Significance of memory properties in prey choice decisions. Ecological Modelling 115, 177-189.

Hofbauer, J., Sigmund, K., 1998. Evolutionary games and population dynamics. Cambridge University Press, Cambridge.

Huxel, G. R., McCann, K., 1998. Food web stability: The influence of trophic flows across habitats. The American Naturalist 152, 460-469.

Krebs, J. R., Erichsen, J. T., Webber, M. I., Charnov, E. L., 1977. Optimal prey selection in the great tit (Parus major). Animal Behaviour 25, 30-38.

Křivan, V., 1996. Optimal foraging and predator-prey dynamics. Theoretical Population Biology 49, 265-290.

Křivan, V., 1997. Dynamic ideal free distribution: Effects of optimal patch choice on predator-prey dynamics. American Naturalist 149, 164-178.

Křivan, V., Cressman, R., 2009. On evolutionary stability in prey-predator models with fast behavioral dynamics. Evolutionary ecology research 11, 227-251.

Křivan, V., Cressman, R., Schneider, C., 2008. The Ideal Free Distribution: A review and synthesis of the game theoretic perspective. Theoretical Population Biology 73, $403-425$.

Křivan, V., Schmitz, O. J., 2003. Adaptive foraging and flexible food web topology. Evolutionary Ecology Research 5, 623-652.

Křivan, V., Sirot, E., 2002. Habitat selection by two competing species in a two-habitat environment. American Naturalist 160, 214-234.

Ma, B., Abrams, P., Brassil, C., 2003. Dynamic versus instantaneous models of diet choice. American Naturalist 162, 668-684. 
MacArthur, R. H., Pianka, E. R., 1966. On optimal use of a patchy environment. American Naturalist 100, 603-609.

Mangel, M., Roitberg, B., 1989. Dynamic information and host acceptance by a tephritid fruit fly. Ecological Entomology 14, 181-189.

Maynard Smith, J., Price, G. R., 1973. The logic of animal conflict. Nature 246, 15-18.

McNamara, J. M., Houston, A. I., 1987. Partial preferences and foraging. Animal Behaviour 35, 1084-1099.

McPeek, M. A., Holt, R. D., 1992. The evolution of dispersal in spatially and temporally varying environments. The American Naturalist 140, 1010-1027.

Morris, D. W., 2003. Shadows of predation: habitat-selecting consumers eclipse competition between coexisting prey. Evolutionary Ecology 17, 393-422.

Padrón, V., Trevisan, M. C., 2006. Environmentally induced dispersal under heterogeneous logistic growth. Mathematical Bioscience 199, 160-174.

Rechten, C., Avery, M., Stevens, A., 1983. Optimal prey selection: why do great tits show partial preferences? Animal Behaviour 31, 576-584.

Stephens, D. W., Krebs, J. R., 1986. Foraging theory. Princeton University Press, Princeton, NJ, USA.

Vincent, T. L., Brown, J. S., 2005. Evolutionary game theory, natural selection and darwinian dynamics. Cambridge University Press, Cambridge, UK. 


\section{Appendix A: Evolutionary stability of the diet choice model}

Fitness function (14) can be written as

$$
W\left(\tilde{u}_{2}, u_{2}\right)=\frac{A \tilde{u}_{2}+B}{C \tilde{u}_{2}+D}
$$

where

$$
\begin{gathered}
A=2 e_{2} h_{1} x_{2} \lambda_{2}, \quad B=2 h_{1} e_{1} L_{1} \lambda_{1}-e_{1} H\left(u_{2}\right)\left(1-\sqrt{1-c\left(u_{2}\right) y}\right), \\
C=2 h_{1} h_{2} x_{2} \lambda_{2}, \quad D=2 h_{1}\left(1+h_{1} L_{1} \lambda_{1}\right)-h_{1} H\left(u_{2}\right)\left(1-\sqrt{1-c\left(u_{2}\right) y}\right), \\
c\left(u_{2}\right)=\frac{4 h_{1} L_{1} \lambda_{1}^{2}}{a_{1}\left(1+h_{1} L_{1} \lambda_{1}+h_{2} x_{2} u_{2} \lambda_{2}\right)^{2}}, \quad H\left(u_{2}\right)=1+h_{1} L_{1} \lambda_{1}+h_{2} x_{2} u_{2} \lambda_{2} .
\end{gathered}
$$

First, I calculate the singular strategy and study its evolutionary stability. The gradient of the fitness function with respect to the mutant strategy

$$
\frac{\partial W}{\partial \tilde{u}_{2}}\left(\tilde{u}_{2}, u_{2}\right)=\frac{A D-B C}{\left(D+C \tilde{u}_{2}\right)^{2}} .
$$

At the singular strategy this gradient when evaluated at $\tilde{u}_{2}=u_{2}$ must be zero, i. e., $A D-B C=0$. When I substitute expressions for $A, B, C, D$ and after some simplification I obtain the following equation

$$
\sqrt{1-\frac{4 h_{1} L_{1} y \lambda_{1}^{2}}{a_{1}\left(1+h_{1} L_{1} \lambda_{1}+h_{2} u_{2} x_{2} \lambda_{2}\right)^{2}}}=1-\frac{2 h_{1}\left(\left(e_{1} h_{2}-e_{2} h_{1}\right) L_{1} \lambda_{1}-e_{2}\right)}{\left(e_{1} h_{2}-e_{2} h_{1}\right)\left(1+h_{1} L_{1} \lambda_{1}+h_{2} u_{2} x_{2} \lambda_{2}\right)} .
$$

This equation posses a solution only provided the right hand side is between 0 and 1 , i. e., when

$$
\frac{e_{2}}{\lambda_{1}\left(e_{1} h_{2}-e_{2} h_{1}\right)}<L_{1}<\frac{e_{2} h_{1}+e_{1} h_{2}+h_{2}\left(e_{1} h_{2}-e_{2} h_{1}\right) u_{2} x_{2} \lambda_{2}}{h_{1}\left(e_{1} h_{2}-e_{2} h_{1}\right) \lambda_{1}} .
$$

Solving equation (18) yields the singular strategy (15). Of course, $u_{2}^{*}$ must be between 0 and 1 which holds for consumer densities that satisfy $y_{1}^{*} \leq y \leq y_{2}^{*}$ where

$$
y_{1}^{*}=\frac{a_{1} e_{1} h_{2}\left(L_{1} \lambda_{1}\left(e_{1} h_{2}-e_{2} h_{1}\right)-e_{2}\right)}{\left(e_{2} h_{1}-e_{1} h_{2}\right)^{2} L_{1} \lambda_{1}^{2}}
$$

and

$$
y_{2}^{*}=\frac{a_{1} h_{2}\left(L_{1} \lambda_{1}\left(e_{1} h_{2}-e_{2} h_{1}\right)-e_{2}\right)\left(e_{1}+\left(e_{1} h_{2}-e_{2} h_{1}\right) x_{2} \lambda_{2}\right)}{\left(e_{2} h_{1}-e_{1} h_{2}\right)^{2} L_{1} \lambda_{1}^{2}} .
$$

If consumer density is too low $\left(y<y_{1}^{*}\right)$, consumers will feed on the more profitable prey type only while at high densities $\left(y>y_{2}^{*}\right)$ they will feed on the alternative prey type upon each encounter. In particular, the carrying capacity for the more profitable prey type must be high enough, i. e.,

$$
L_{1}>\frac{e_{2}}{\lambda_{1}\left(e_{1} h_{2}-e_{2} h_{1}\right)}
$$


for consumer partial preferences to arise. If the opposite inequality holds, fitness maximizes at $u_{2}=1$. I derived the singular strategy under the assumption that the right hand side of equation (18) is positive, because otherwise no singular solution exists. Substituting the singular strategy to the right hand side of (18) leads to expression

$$
\frac{\left(e_{2} h_{1}-e_{1} h_{2}\right)^{2} L_{1} y \lambda_{1}^{2}-a_{1} h_{1}\left(e_{2}+e_{2} h_{1} L_{1} \lambda_{1}-e_{1} h_{2} L_{1} \lambda_{1}\right)^{2}}{\left(e_{2} h_{1}-e_{1} h_{2}\right)^{2} L_{1} y \lambda_{1}^{2}+a_{1} h_{1}\left(e_{2}+e_{2} h_{1} L_{1} \lambda_{1}-e_{1} h_{2} L_{1} \lambda_{1}\right)^{2}}
$$

that is positive provided

$$
y>\frac{a_{1} h_{1}\left(e_{2}+e_{2} h_{1} L_{1} \lambda_{1}-e_{1} h_{2} L_{1} \lambda_{1}\right)^{2}}{\left(e_{2} h_{1}-e_{1} h_{2}\right)^{2} L_{1} \lambda_{1}^{2}} .
$$

This condition must hold together with other constraints for the singular strategy to be between 0 and 1 .

Because $A D-B C=0$ at the singular strategy, $W\left(\tilde{u}_{2}, u_{2}^{*}\right)$ is independent of the mutant strategy $\tilde{u}_{2}$ and, after some calculations, $W\left(\tilde{u}_{2}, u_{2}^{*}\right)=e_{2} / h_{2}$ for all mutant strategies $u_{2}$.

To study evolutionary stability of the singular strategy I need to check the local stability condition (Hofbauer and Sigmund, 1998) that asserts that mutants cannot spread in the resident population. The local stability condition requires that

$$
W\left(u_{2}^{*}, u_{2}\right)>W\left(u_{2}, u_{2}\right)
$$

for every $u_{2} \neq u_{2}^{*}$ in a neighborhood of the singular strategy $u_{2}^{*}$. Let $g\left(u_{2}\right)=W\left(u_{2}^{*}, u_{2}\right)-$ $W\left(u_{2}, u_{2}\right)$. Thus, $g\left(u_{2}^{*}\right)=0$ and with a help of some computer algebra package (e. g., Mathematica) it is easy to show that provided inequality (19) holds, $g^{\prime}\left(u_{2}^{*}\right)=0$ and

$g^{\prime \prime}\left(u_{2}^{*}\right)=\frac{2 a_{1}^{3} h_{2} x_{2}^{2}\left(e_{2}+\left(e_{2} h_{1}-e_{1} h_{2}\right) L_{1} \lambda_{1}\right)^{4} \lambda_{2}^{2}}{\left(e_{1} h_{2}-e_{2} h_{1}\right) L_{1}^{2} y^{2} \lambda_{1}^{4}\left(\left(e_{2} h_{1}-e_{1} h_{2}\right)^{2} L_{1} y \lambda_{1}^{2}-a_{1} h_{1}\left(e_{2}+\left(e_{2} h_{1}-e_{1} h_{2}\right) L_{1} \lambda_{1}\right)^{2}\right)}>0$.

Thus, $g\left(u_{2}\right)>0$ in a neighborhood of the singular strategy $\left(u_{2} \neq u_{2}^{*}\right)$ and the local ESS condition (20) holds.

Second, I will prove that the singular strategy is also continuously stable. This follows from the fact (Eshel, 1983) that

$$
\begin{gathered}
\frac{\partial^{2} W\left(u_{2}^{*}, u_{2}^{*}\right)}{\partial \tilde{u}_{2}^{2}}+\frac{\partial^{2} W\left(u_{2}^{*}, u_{2}^{*}\right)}{\partial \tilde{u}_{2} \partial u_{2}}= \\
\frac{a_{1}^{3} h_{2} x_{2}^{2}\left(e_{2}+e_{2} h_{1} L_{1} \lambda_{1}-e_{1} h_{2} L_{1} \lambda_{1}\right)^{4} \lambda_{2}^{2}}{\left(e_{1} h_{2}-e_{2} h_{1}\right) L_{1}^{2} y^{2} \lambda_{1}^{4}\left(-\left(e_{2} h_{1}-e_{1} h_{2}\right)^{2} L_{1} y \lambda_{1}^{2}+a_{1} h_{1}\left(e_{2}+e_{2} h_{1} L_{1} \lambda_{1}-e_{1} h_{2} L_{1} \lambda_{1}\right)^{2}\right)}<0
\end{gathered}
$$

for consumer densities that satisfy (19). 


\section{Appendix B: Derivation of model (17).}

Substituting $x_{1+}$ given by (13) in the right hand side of the consumer population growth equation (11) leads to

$$
\frac{d y}{d t}=\frac{y\left(A+\left(e_{1}-h_{1} m\right) \sqrt{-4 h_{1} L_{1} y \lambda_{1}^{2}+a_{1} H^{2}}\right.}{h_{1}\left(\sqrt{a_{1}} H+\sqrt{-4 h_{1} L_{1} y \lambda_{1}^{2}+a_{1} H^{2}}\right)}
$$

where

$$
\begin{gathered}
A=\sqrt{a_{1}}\left(-e_{1}-h_{1} m+e_{1} h_{1} L_{1} \lambda_{1}-h_{1}^{2} L_{1} m \lambda_{1}+\left(2 e_{2} h_{1}-h_{2}\left(e_{1}+h_{1} m\right)\right) u_{2} x_{2} \lambda_{2}\right), \\
H=1+h_{1} L_{1} \lambda_{1}+h_{2} x_{2} u_{2} \lambda_{2} .
\end{gathered}
$$

Extending the above fraction by expression $h_{1}\left(\sqrt{a_{1}} H-\sqrt{-4 h_{1} L_{1} y \lambda_{1}^{2}+a_{1} H^{2}}\right)$ leads to

$$
\begin{gathered}
\frac{d y}{d t}=\frac{\left(e_{1}-h_{1} m\right) y}{h_{1}}+\frac{\sqrt{a_{1}}\left(u_{2} x_{2} \lambda_{2}\left(e_{2} h_{1}-e_{1} h_{2}\right)-e_{1}\right)\left(\sqrt{a_{1}} H-\sqrt{a_{1} H^{2}-4 h_{1} L_{1} y \lambda_{1}^{2}}\right)}{2 h_{1}^{2} L_{1} \lambda_{1}^{2}} \\
=\frac{\left(e_{1}-h_{1} m\right) y}{h_{1}}-\frac{2 \sqrt{a_{1}} y\left(e_{1}+u_{2} x_{2} \lambda_{2}\left(e_{1} h_{2}-e_{2} h_{1}\right)\right)}{h_{1}\left(\sqrt{a_{1}} H+\sqrt{a_{1} H^{2}-4 h_{1} L_{1} y \lambda_{1}^{2}}\right)} \\
=\frac{\left(e_{1}-h_{1} m\right) y}{h_{1}}\left(1-\frac{2 h_{1} \sqrt{a_{1}}\left(e_{1}+u_{2} x_{2} \lambda_{2}\left(e_{1} h_{2}-e_{2} h_{1}\right)\right)}{\left(e_{1}-h_{1} m\right) h_{1} \sqrt{a_{1}} H\left(1+\sqrt{1-4 h_{1} L_{1} y \lambda_{1}^{2} /\left(a_{1} H^{2}\right)}\right)}\right) \\
=r y\left(1-\frac{K}{1+\sqrt{1-c y}}\right)
\end{gathered}
$$

where $r=\frac{e_{1}}{h_{1}}-m, K=2 \frac{e_{1}+u_{2} \lambda_{2} x_{2}\left(e_{1} h_{2}-e_{2} h_{1}\right)}{\left(e_{1}-h_{1} m\right) H}$ and $c=\frac{4 h_{1} L_{1} \lambda_{1}^{2}}{a_{1} H^{2}}$. There exists a non-zero equilibrium of the single species consumer model (21)

$y^{*}=\frac{K(2-K)}{c}=\frac{a_{1}\left(e_{1}+\left(e_{1} h_{2}-e_{2} h_{1}\right) u_{2} x_{2} \lambda_{2}\right)\left(L_{1}\left(e_{1}-h_{1} m\right) \lambda_{1}-m+\left(e_{2}-h_{2} m\right) u_{2} x_{2} \lambda_{2}\right)}{L_{1} \lambda_{1}^{2}\left(e_{1}-h_{1} m\right)^{2}}$

which is exactly the same as the interior consumer equilibrium of model (11). For $y^{*}$ to be an equilibrium, it must be positive, i. e., $0<K<2$. This condition is equivalent to

$$
h_{1}<e_{1} / m, \quad L_{1}>\frac{m-\left(e_{2}-h_{2} m\right) u_{2} x_{2} \lambda_{2}}{\lambda_{1}\left(e_{1}-m h_{1}\right)} .
$$

Equilibrium $y^{*}$ is asymptotically stable when the sign of the derivative of the right hand side of (21) evaluated at this equilibrium

$$
J=\left.\frac{d}{d y}\left(r y\left(1-\frac{K}{1+\sqrt{1-c y}}\right)\right)\right|_{y=y^{*}}=\frac{r(K-2)}{2(K-1)}
$$


is negative, i. e., $1<K<2$. I remark that condition $1<K$ is equivalent to

$$
L_{1}<\frac{e_{1}+h_{1} m+\left(-2 e_{2} h_{1}+h_{2}\left(e_{1}+h_{1} m\right)\right) u_{2} x_{2} \lambda_{2}}{h_{1}\left(e_{1}-h_{1} m\right) \lambda_{1}}
$$

which is the condition for the interior equilibrium of the resource-consumer model (11), to be stable. For larger values of the environmental carrying capacities the interior equilibrium is unstable and a stable limit cycle arises in the Rosenzweig-MacArthur model (11) (Hofbauer and Sigmund, 1998).

The above analysis assumed fixed consumer preference for the alternative resource. Now I will study population dynamics (17) driven by the singular strategy. Substituting singular strategy (15) in population dynamics (17) I get

$$
\frac{d y}{d t}=y \frac{\left(e_{2}-h_{2} m\right)}{h_{2}}
$$

for consumer densities satisfying $y_{1}^{*}<y(t)<y_{2}^{*}$ and (19). For $h_{2}<e_{2} / m$ consumer densities increase, while for larger handling times they decrease. No nontrivial equilibrium exists. If $y \geq y_{2}^{*}$ then $u_{2}=1$ and population dynamics on the stable manifold are obtained by substituting $u_{2}=1$ to (17). These population dynamics have equilibrium

$$
y_{e q}^{1}=\frac{a_{1}\left(e_{1}+\left(e_{1} h_{2}-e_{2} h_{1}\right) x_{2} \lambda_{2}\right)\left(e_{1} L_{1} \lambda_{1}+e_{2} x_{2} \lambda_{2}-m\left(1+h_{1} L_{1} \lambda_{1}+h_{2} x_{2} \lambda_{2}\right)\right)}{L_{1}\left(e_{1}-h_{1} m\right)^{2} \lambda_{1}^{2}} .
$$

Similarly when $y<y_{1}^{*}, u_{2}=0$ and population dynamics on the stable manifold are obtained by substituting $u_{2}=0$ to (17). These population dynamics have equilibrium

$$
y_{e q}^{0}=\frac{a_{1} e_{1}\left(e_{1} L_{1} \lambda_{1}-m\left(1+h_{1} L_{1} \lambda_{1}\right)\right)}{L_{1}\left(e_{1}-h_{1} m\right)^{2} \lambda_{1}^{2}} .
$$


Figures caption.

Figure 1: Solutions of the patch model (3) (solid line) where consumers follow the optimal foraging strategy given by (2), and solutions of the single-species model (7)(dashed line). Resource densities for model (7) are given by $x_{i}=L_{i}\left(1-y(t) \lambda_{i} v_{i} / a_{i}\right)$ with the optimal strategy $v_{i}$ given by (6). The left panel (A,B,C) assumes that consumer demographic parameters $\left(e_{1}=0.015, e_{2}=0.01, m_{1}=m_{2}=0.02\right)$ are much smaller when compared to resource parameters. This discrepancy causes resource population dynamics to run on a fast time scale when compared with consumer population dynamics. The right panel (D,E,F) assumes more similar time scales for both resource and consumer dynamics $\left(e_{1}=0.15, e_{2}=0.1, m_{1}=m_{2}=0.2\right)$. Other parameters used in simulations: $L_{1}=L_{2}=10, a_{1}=1.5, a_{2}=0.5, \lambda_{1}=\lambda_{2}=1$.

Figure 2: Resource isocline of model (11). Only the solid part of the isocline is stable provided resource 1 population dynamics are fast when compared to consumer population dynamics. Arrows indicate direction of trajectories.

Figure 3: Panel A shows consumer preference (15) for the alternative prey type in the diet choice model as a function of consumer density. Panel B compares a trajectory of the resource-consumer model (11) (dashed lines) where consumers follow predictions of the classical prey model (i. e., zero-one rule) with a trajectory of the reduced model (17) (solid lines). The corresponding trajectory for resources is given by (13). Panel C shows the corresponding consumer optimal strategy. Panel D shows the solid trajectory from panel $\mathrm{B}$ in the resource 1-consumer preference-consumer density phase space. Parameters: $a_{1}=1.2, h_{1}=h_{2}=0.2, e_{1}=0.2, e_{2}=0.05, \lambda_{1}=\lambda_{2}=0.5, x_{2}=8$, $m=0.2, L_{1}=10$. 

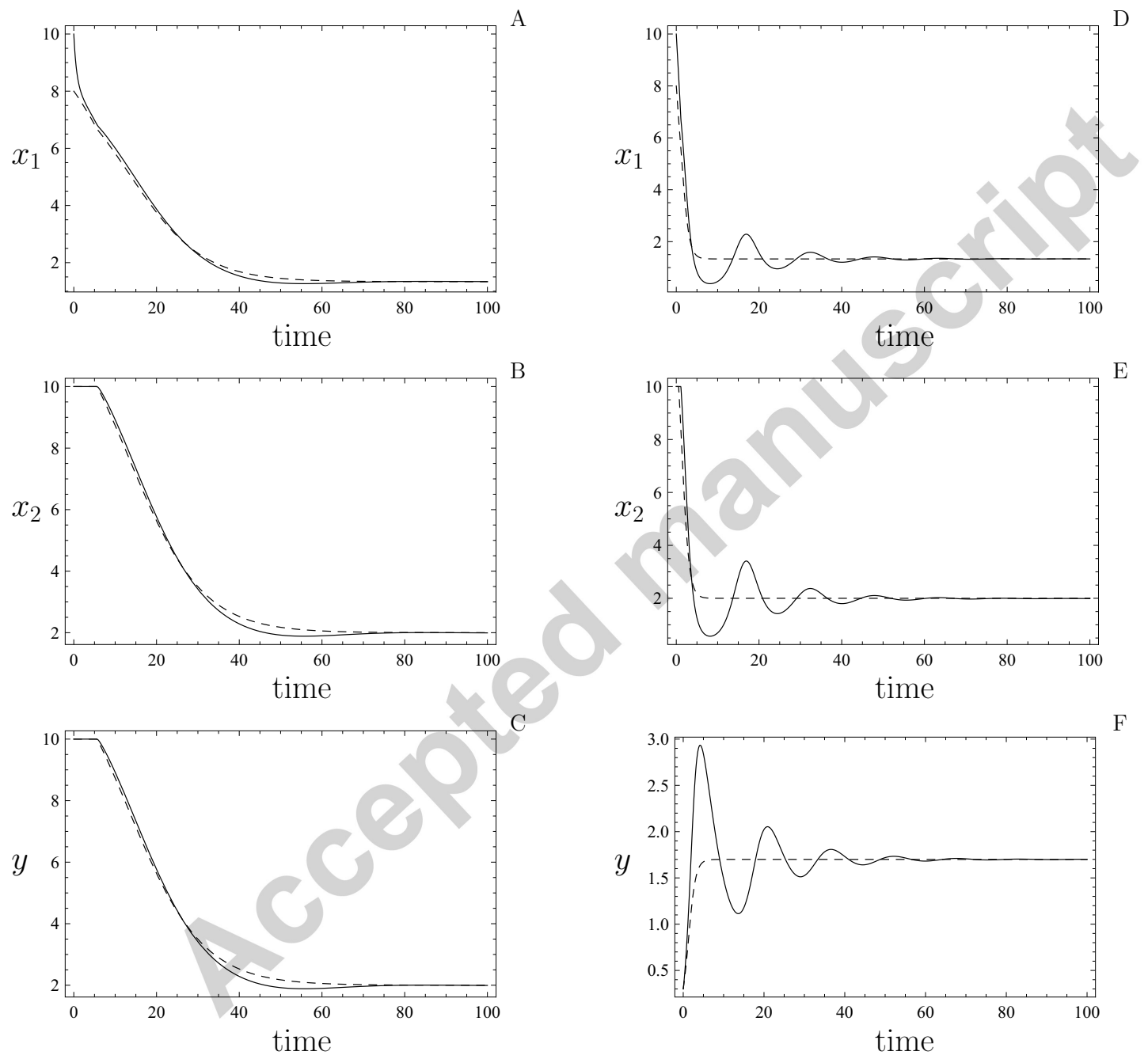

Figure 1: 


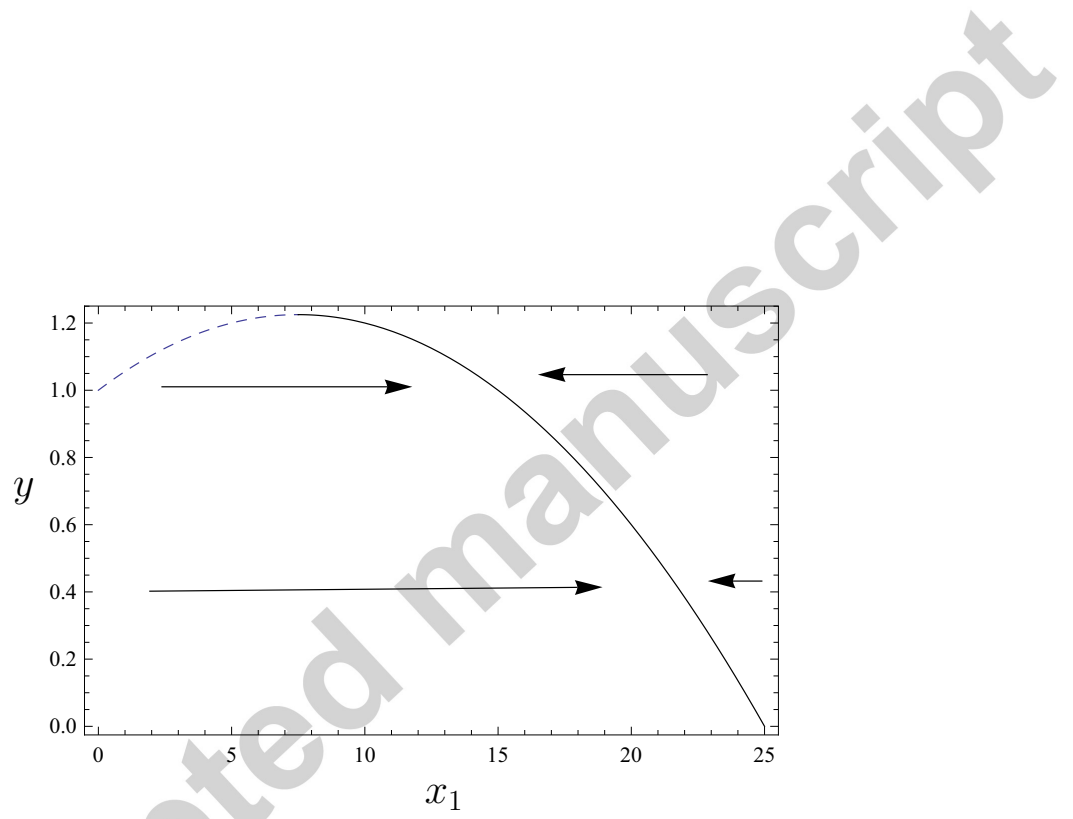

Figure 2: 

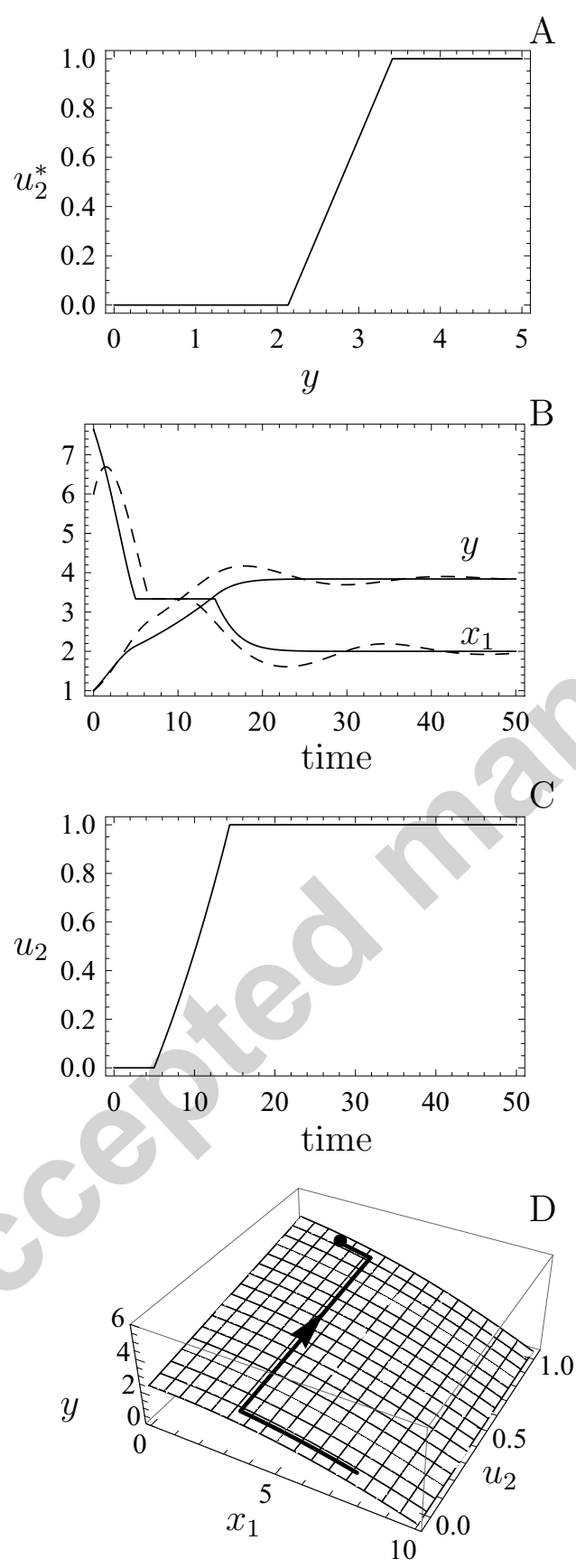

Figure 3: 